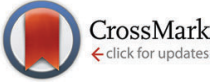

Cite this: Phys. Chem. Chem. Phys., 2015, 17, 13915

Received 26th March 2015, Accepted 30th April 2015

DOI: $10.1039 / c 5 c p 01779 c$

www.rsc.org/pccp

\title{
A new approach to elucidating repair reactions of resveratrol $\dagger$
}

\author{
Christoph Kerzig, Selgar Henkel and Martin Goez*
}

\begin{abstract}
The repair by co-antioxidants of the phenoxy radical of resveratrol, the famous health-preserving ingredient of red wine, is a key step of radical scavenging cascades in nature. To generate that radical, we employed $355 \mathrm{~nm}$ photoionization as a direct and selective access that reduces the chemical complexity and is equally applicable in organized phases; to monitor it, we used its hitherto unreported absorption in the red where no other species in our systems interfere. With this novel approach, we measured rate constants and H/D kinetic isotope effects for the repairs by ascorbate, trolox (a vitamin $\mathrm{E}$ analogue) and 4-aminophenol, and identified the mechanisms as one-step hydrogen abstractions. Cysteine and glutathione are unreactive. In micellar solution (SDS), the repair by ascorbate is much slower and involves only the hydrophilic phenoxy moieties protruding from the micelles. The new experimental strategy also led to a reevaluation of extinction coefficients, rate constants and mechanisms.
\end{abstract}

\section{Introduction}

Resveratrol (ResOH; for the formula, see Fig. 1b) is a polyphenol ingredient of red wine with remarkable health-preserving properties: not only is this powerful antioxidant thought to be responsible for the "French paradox"1_a low mortality from coronary heart diseases despite a high uptake of unsaturated fats-but it also exhibits anticancer ${ }^{2}$ and antiaging ${ }^{3}$ effects. This explains why it keeps attracting immense attention, culminating in 1700 new publications in 2013 alone as referenced by SciFinder ${ }^{\circledR}$.

Previous studies of ResOH as an antioxidant have focused almost exclusively on its radical scavenging. ${ }^{4-18}$ In contrast, extremely little is known about its subsequent regeneration from the resulting phenoxy radicals $\mathbf{R e s}^{\bullet}$ by co-antioxidants, although these repair reactions are of similar importance because these integral parts of the radical scavenging cascades in nature ${ }^{19}$ increase the biological efficiency of ResOH, so reduce the necessary nutritional uptake. ${ }^{20}$ Spin-trap experiments ${ }^{21}$ and steady-state absorption spectroscopy ${ }^{22}$ gave qualitative evidence for such a repair by ascorbate or glutathione, and a rate constant for the regeneration by 4 -aminophenol has been mentioned in passing. ${ }^{23}$ This paucity of information-as opposed to the situation for other phenolic antioxidants, in

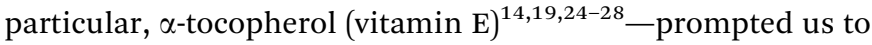
investigate the reactions of $\mathbf{R e s O}^{\bullet}$ with typical co-antioxidants mechanistically and kinetically.

Martin-Luther-Universität Halle-Wittenberg, Institut für Chemie, Kurt-Mothes-Str. 2, D-06120 Halle (Saale), Germany.E-mail: martin.goez@chemie.uni-halle.de $\dagger$ Electronic supplementary information (ESI) available. See DOI: 10.1039/ c5cp01779c
For this purpose, the usual access to $\mathbf{R e s O}^{\bullet}$ through the scavenging of other radicals $\mathbf{R}^{\bullet}$ by $\mathbf{R e s} \mathbf{O H}$ possesses two intrinsic drawbacks. First, the systems become needlessly complex because different resveratrol-derived radicals can be formed in parallel, ${ }^{13,15,16}$ interconvert, ${ }^{16}$ and decay by different pathways; ${ }^{13,15}$ because $\mathbf{R}^{\bullet}$ can also be scavenged by the co-antioxidant; ${ }^{22}$ and because $\mathbf{R e s O}^{\bullet}$ is the intermediate of a consecutive reaction. Second, additional complications arise in microheterogeneous systems because this method for producing $\mathbf{R e s O}^{\bullet}$ relies on a bimolecular step, which is strongly influenced by the partitioning of $\mathbf{R}^{\bullet}$ (or its precursors) and $\mathbf{R e s O H}$ between the microphases.

In the present work, we have avoided all these difficulties by generating $\mathbf{R e s O}^{\bullet}$ in a monomolecular process direct from ResOH using nanosecond laser flash photolysis. For relevant ground-state properties of ResOH, see Section 2.1 of the ESI $\dagger$ (hereafter abbreviated as SI-2.1, ESI $\dagger$ etc.). There is ample precedent for the photoionization of phenolic compounds with UV-B and UV-C light, ${ }^{25,27-33}$ but these wavelengths are also strongly absorbed by most co-antioxidants, which are normally present in higher concentrations than is ResOH, so filter effects and side reactions are inevitable. The present study exploits the bathochromic shifts by the longer conjugated systems in ResOH and its radical: as we will show, in aqueous medium the near-UV (355 nm) photoionization of ResOH, which has recently been thoroughly investigated in acetonitrile, $^{34}$ affords ResO $^{\bullet}$ in amply sufficient yield for spectrophotometric monitoring of its further fate with the additional advantage that not only the excitation but also the detection, for which we use a hitherto unreported band of $\mathbf{R e s O}^{\bullet}$ in the red, fall into a spectral window where the co-antioxidants do not absorb. 


\section{Results and discussion}

Laser flash photolysis (355 nm) of ResOH causes photoionization also in aqueous solution at $\mathrm{pH} 6.5$, as is established by the characteristic absorption of the hydrated electron $\mathrm{e}_{\mathrm{aq}}{ }^{--}$ and the complete blanking of that absorption upon saturation with the selective electron scavenger $\mathrm{N}_{2} \mathrm{O}$ (details, covering also the signal separation procedures, are given in SI-1, ESI $\dagger$ ). The remaining transient absorptions are displayed in Fig. 1a; in addition to a strong band centered at $410 \mathrm{~nm}$, there is a weaker, structured one with maxima at 650 and $720 \mathrm{~nm}$. Not only are the intensities of these three peaks strictly proportional to one another, but immediately after the laser flash they are also proportional to the absorption of $\mathrm{e}_{\mathrm{aq}}{ }^{-{ }^{-}}$(inset of the figure). As is evident from these observations, the spectrum of Fig. 1a is due to a single intermediate, and the latter is formed from the same excited state that ejects the electron.

That intermediate can only be ResO $^{*}$ because the photoionization of ResOH initially affords the radical cation $\mathbf{R e s O H}^{\bullet+}$, which is known to be instantaneously deprotonated by water $\left(k=1 \times 10^{8} \mathrm{M}^{-1} \mathrm{~s}^{-1}\right.$ in wet acetonitrile, corresponding to a conversion into $\mathbf{R e s}^{\bullet}$ on a subnanosecond timescale in our medium) $;{ }^{34}$ besides, shape and position of the dominant peak perfectly agree with the literature. ${ }^{15,23,34}$ For the spectral calibration by three independent methods, which all gave the same-but twice as high as previously reported ${ }^{34}$-extinction coefficient $\varepsilon\left(\right.$ ResO $\left.^{*}\right)$ at $410 \mathrm{~nm}$, see SI 2.2.2 (ESI $\dagger$ ). We stress that none of our results below depends in any way on the actual value of $\varepsilon\left(\operatorname{Res}^{\bullet}\right)$.

The spectral feature in the red, which might have been overlooked in earlier studies for sensitivity reasons, is reproduced by quantum-mechanical calculations (the two strongest computed transitions are also shown in Fig. 1a; further details can be found in SI-2.2.1, ESI $\dagger$ ). Although that band is much weaker than the one at $410 \mathrm{~nm}$, it has the advantage of allowing a direct monitoring of the ResO $\mathbf{O}^{\bullet}$ concentration because all our co-antioxidants and their radicals are completely transparent in that region, whereas these radicals (except in the ascorbate case) strongly contribute to the absorbance observed at $410 \mathrm{~nm}$. The detection in the red does not adversely affect the sensitivity, as the very good signal-to-noise ratio (compare, Fig. 2b) shows.

The upward curvature in the low-intensity regime of the electron yield as function of the excitation energy (Fig. 1b) clearly identifies the ionization as biphotonic. ${ }^{35}$ We found no evidence whatsoever for the occurrence of a triplet state in the reaction, so the first excited singlet state $S_{1}$ of ResOH must absorb the second photon. Stern-Volmer experiments with $\mathrm{I}^{-}$as quencher gave a lifetime of $36 \mathrm{ps}$ for $\mathrm{S}_{1}$ (see, SI-2.1, ESI $\dagger$ ). All these findings are consistent with the results of femtosecond experiments in acetonitrile. ${ }^{34}$ Because the $S_{1}$ lifetime is much shorter than the duration of our laser flashes (5 ns), the intensity dependence is accurately describable with the approximation of a fast preequilibrium between ground state and $\mathrm{S}_{1}$; this leads to a two-parameter model recently derived by us, ${ }^{36}$ which is not only applicable to low intensities but valid throughout the whole turnover range. As is estimated (SI-3 ESI, $\uparrow$ where the effect of a geometrical isomerization is also discussed) from the model and its best-fit parameters in the experiment of Fig. $1 \mathrm{~b}$, the quantum yield of ionization of $\mathrm{S}_{1}$ by the second photon must be quite high, which explains why a substantial amount of ResO $^{\bullet}$ can be produced at $355 \mathrm{~nm}$ despite the short $\mathrm{S}_{1}$ life and low ground-state extinction coefficient $\varepsilon(\mathbf{R e s} \mathbf{O H})$. Owing to this property, the electron ejection from ResOH thus provides a convenient access to ResO ${ }^{\bullet}$ with this wavelength, where none of our co-antioxidants is photoionizable.

Also included in Fig. 1b is the outcome of the same experiment in sodium dodecyl sulfate (SDS) micelles. As the best-fit parameters show (SI-3, ESI $\dagger$ ), the noticeably higher electron
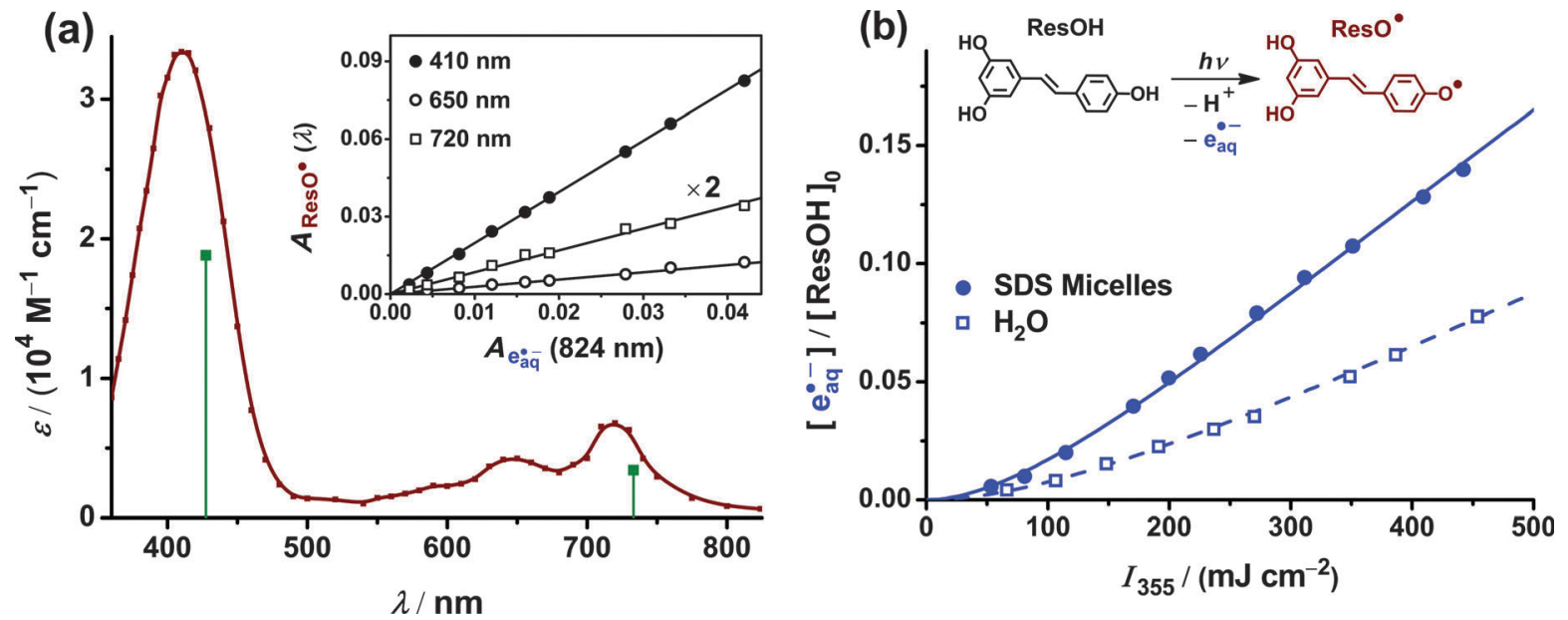

Fig. 1 Near-UV (355 nm) photoionization of an aqueous solution of $8 \times 10^{-5} \mathrm{M}$ ResOH ([ResOH $]_{0}$ ) at pH 6.5. (a) Absorption spectrum of ResO• obtained by $355 \mathrm{~nm}$ laser flash photolysis $\left(349 \mathrm{~mJ} \mathrm{~cm}^{-2}\right.$ ). SI-2.2.2 (ESI $\dagger$ ) enlarges on the spectral calibration. Green vertical lines indicate calculated transitions (TD-DFT, BLYP/6-311++G(2d,2p)); full particulars can be found in SI-2.2.1 (ESI $\dagger$ ). Inset, ResO• peak absorptions against the $\mathrm{e}_{\mathrm{aq}}{ }^{--}$absorption at a reference wavelength (see, $\mathrm{SI}-1, \mathrm{ESI} \dagger$ ) at different laser intensities. (b) Intensity dependence of $\mathrm{e}_{\mathrm{aq}}{ }^{{ }^{-}-}$formation relative to the substrate concentration $[\mathrm{ResOH}]_{0}$ in aqueous solution (open squares and dashed line) and in SDS (50 mM) micelles (filled circles and solid line); fit functions and best-fit parameters are discussed in $\mathrm{SI}-3$ (ESI†). For further details, see text. 

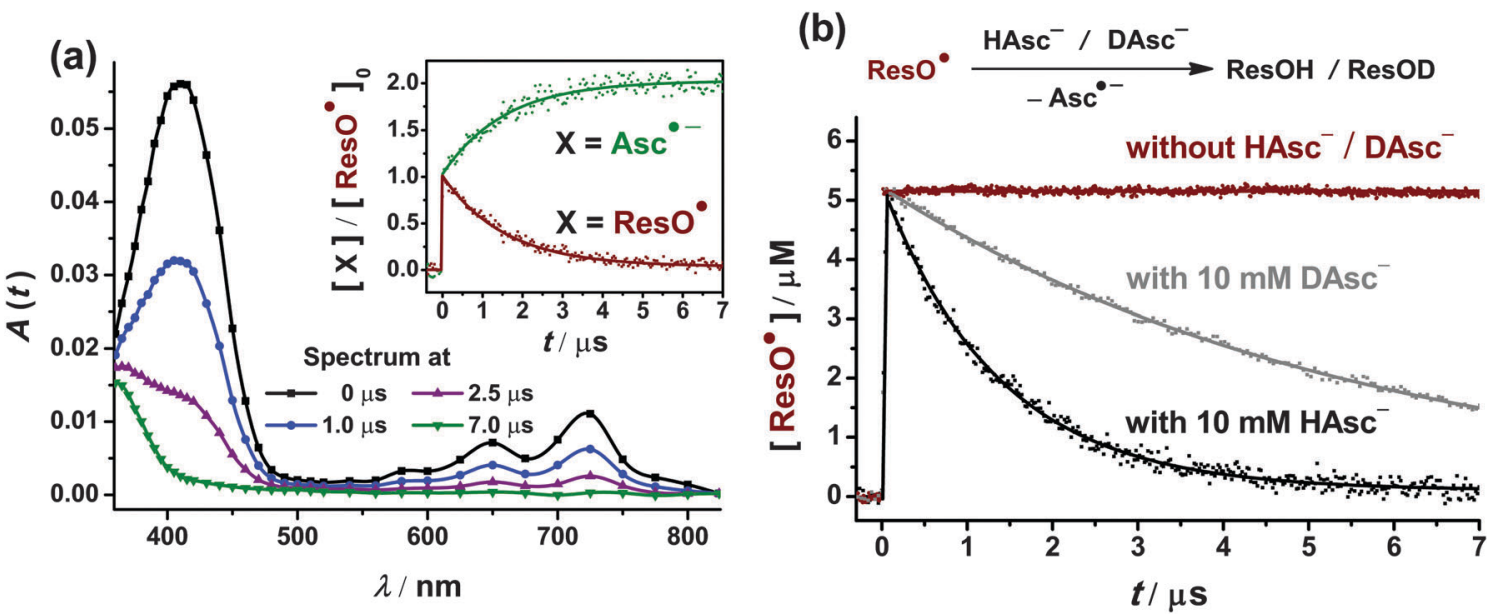

Fig. 2 Repair reaction of ResO• by $10 \mathrm{mM}$ ascorbate at $\mathrm{pH}$ 6.5. ResO• was selectively produced by near-UV photoionization (355 $\mathrm{nm}, 389 \mathrm{~mJ} \mathrm{~cm}^{-2}$ ) of an $8 \times 10^{-5} \mathrm{M} \mathrm{N}_{2} \mathrm{O}$-saturated solution of ResOH. (a) Main plot, transient absorption spectra at different times after the laser flash. Inset, time dependence

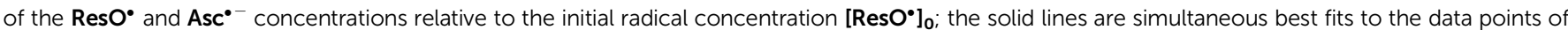
$\exp (-k t)$ and $2-\exp (-k t)$, respectively, with $k$ being identical. (b) ResO• decay without ascorbate, with protiated ascorbate, and with deuterated ascorbate; the fit functions (solid lines) of the last two are given by $\left[\text { ResO } 0^{*}\right]_{0} \times \exp (-k t)$. Table 1 lists the rate constants $k$. For details, see text and SI-2.2.3 (ESI $\dagger$ ).

yield in this medium must largely be ascribed to an increase of $\varepsilon(\mathbf{R e s O H})$ caused by a slight red shift of the ground-state spectrum. The figure demonstrates that the photoionization route to $\mathbf{R e s O}^{\bullet}$ is equally viable in a microheterogeneous system.

Fig. 2 sums up our experimental results obtained, with this new approach, for the repair reaction of $\mathbf{R e s O}^{\bullet}$ by a typical co-antioxidant, the ascorbate monoanion $\mathbf{H A s c}^{-}$. Whereas ResO $^{-}$alone is stable for many microseconds (Fig. 2b and SI-2.2.3, ESI $\dagger$ ), its characteristic absorptions decay rapidly in the presence of $10 \mathrm{mM}$ of $\mathbf{H A s c}^{-}$and a new band centered at $360 \mathrm{~nm}$ arises (Fig. 2a). The latter is the only further absorption, as is revealed by subtracting an appropriately scaled spectrum of pure $\mathbf{R e s O}^{\circ}$, and that remainder is identical with the well-known spectrum of the extremely long-lived radical $\mathbf{A s c}^{\bullet-} \cdot .^{37,38}$ This facile separation at any point of time after the laser flash is possible because there are no competing absorptions from $\mathbf{A s c}^{\bullet-}$ nor from the radicals of our other co-antioxidants in the region of the reference peaks of ResO' at 650 or $720 \mathrm{~nm}(\mathrm{SI}-4, \mathrm{ESI} \dagger)$.

The inset of Fig. 2a compares the time dependences of the $\mathbf{R e s O}^{\bullet}$ and $\mathbf{A s c}^{\bullet-}$ concentrations extracted (with the extinction coefficients given in SI-2.2.2 and SI-4, ESI $\dagger$ ) from the separated kinetic spectra. The decrease of $\mathbf{R e s O}^{\bullet}$ and the increase of $\mathbf{A s c}^{\bullet-}$ are found to be first-order processes with exactly the same rate, so the experiment evidently captures the repair reaction displayed at the top of Fig. $2 \mathrm{~b}$. As a side issue, that gradual rise of the $\mathbf{A s c}^{\mathbf{*}}{ }^{-}$concentration is superimposed on a constant floor that is reached immediately after the laser flash and equals the ResO' concentration at that very moment; its origin is not a photoionization of $\mathbf{H A s c}^{-}$, which is impossible at our excitation wavelength, ${ }^{38}$ but a sequence of two quasi-instantaneous scavenging reactions starting from the stoichiometrically formed $\mathrm{ResO}^{\boldsymbol{\bullet}}$ by-product $\mathrm{e}_{\mathrm{aq}}{ }^{\boldsymbol{}}$ (SI-1 and SI-2.2.2, ESI†). After correction for this floor, not only the rate constants but also the amplitudes of the ResO' decrease and the $\mathbf{A s c}^{\bullet-}$ increase are seen to be identical, which clearly shows that the repair is not accompanied by side reactions.
A variation of the $\mathbf{H A s c}^{-}$concentration confirmed that the co-antioxidant is the repairing agent. The obtained secondorder rate constant, on the other hand, depended neither on the HAsc $^{-}$concentration nor on the laser intensity, showing that radical-radical recombinations cannot compete with the repair. The gross reaction is a hydrogen abstraction, for which a one-step process (either through a direct abstraction or a coupled electron-proton transfer ${ }^{27}$ ) and a two-step process through rate-limiting electron transfer to give $\mathbf{R e s O}^{-}$and $\mathbf{H A s c}^{\circ}$ followed by fast reprotonation of the former and deprotonation of the latter by the solvent would be equally feasible thermodynamically (compare, Table 1). To resolve that mechanistic ambiguity, we measured the repair rate in $\mathrm{D}_{2} \mathrm{O}$, where all hydroxylic protons of both substrate and co-antioxidant are replaced by deuterium. The large H/D kinetic isotope effect (KIE) of 3.9, which is immediately perceived in Fig. 2b, can only be reconciled with the one-step process. This finding agrees very well with that for the repair of the $\alpha$-tocopherol phenoxy radical by $\mathbf{H A s c}^{-}$, where the activation parameters substantiate a concerted electron-proton transfer. ${ }^{27}$

Carrying out the repair in micellar solutions of SDS decreased the rate constant by a factor of 70 (Table 1). Because the negatively charged HAsc $^{-}$is not lipophilic, it can only react

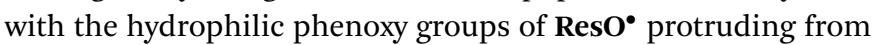
the micelles or with ResO $^{\bullet}$ altogether present in the aqueous phase. When we increased the amount of SDS such as to nearly quadruple the micelle-to-radical concentration ratio, thus modifying the amounts of solubilized and free $\mathbf{R e s O}^{\bullet}$ accordingly, we did not observe any effect on the kinetics, which clearly substantiates the first alternative. Compared to the repair of the $\alpha$-tocopherol phenoxy radical by $\mathbf{H A s c}^{-},{ }^{27}$ the reduction of the reaction rate by the SDS micelle is only a quarter as large in our system, which indicates that the radical moiety of solubilized ResO' is more exposed to the aqueous phase; this is also in accordance with the fact that the repair of 
Table 1 Repair reaction of ResO $0^{\bullet}$ with typical co-antioxidants at $\mathrm{pH} 6.5$

\begin{tabular}{llllll}
\hline Co-antioxidant & Conditions & $k /\left(\mathrm{M}^{-1} \mathrm{~s}^{-1}\right)$ & $\mathrm{KIE}^{a}$ & $-\Delta E\left(\mathrm{HAbs}^{b} /\left(\mathrm{kJ} \mathrm{mol}^{-1}\right)\right.$ & $\left.-\Delta G(\mathrm{ET})^{c} /(\mathrm{kJ} \mathrm{mol})^{-1}\right)$ \\
\hline Ascorbate & Water & $7.1 \times 10^{7}$ & 3.9 & $42^{40}$ & \\
& $30 \mathrm{mM}$ SDS & $1.1 \times 10^{6}$ & & \\
& $90 \mathrm{mM}$ SDS & $1.0 \times 10^{6}$ & & $27^{42}$ & $22^{41}$ \\
4-Aminophenol & Water & $1.4 \times 10^{8}$ & 2.2 & $23^{43 d}$ & $-15^{41}$ \\
Trolox & Water & $1.1 \times 10^{8}$ & 3.1 & $-3^{44}$ & $-44^{41}$ \\
Cysteine & Water & No reaction & n/a &
\end{tabular}

${ }^{a}$ H/D kinetic isotope effect. ${ }^{b}$ The energetics of the direct hydrogen abstraction (HAbs) were obtained from the difference of experimental bond dissociation energies of ResOH (350 kJ mol$\left.{ }^{-1}\right)^{45}$ and the co-antioxidants (references given at the table entry). ${ }^{c}$ The Gibbs energies of electron transfer (ET) were obtained from the difference of oxidation potentials of ResOH at pH $6.5\left(0.64 \mathrm{~V} v s\right.$. NHE) ${ }^{46}$ and the co-antioxidants (references given at the table entry). ${ }^{d}$ Value for $\alpha$-tocopherol.

ResO $^{\bullet}$ by HAsc $^{-}$in that model membrane is about 20 times faster than that of the $\alpha$-tocopherol phenoxy radical.

We next extended our studies to 4-aminophenol, which is not a naturally occurring antioxidant but the only reductant for which a rate constant of the reaction with $\mathbf{R e s O}^{\bullet}$ was available,$^{23}$ although without any details on how this was measured. The rate constant obtained with our direct method (Table 1) is 6 times higher than the reported value. The aminophenol also reacts about twice as fast with $\mathbf{R e s O}^{\bullet}$ as does HAsc $^{-}$despite a significantly smaller thermodynamic driving force. Together with the much lower KIE, this might indicate a mechanistic change to an indirect hydrogen abstraction, which would be in accordance with the previous interpretation of that reaction as an electron transfer. ${ }^{23}$ However, $\pi$ stacking with concomitant nonlinearity of the transition state has been invoked as an explanation of an even smaller KIE in a direct hydrogen abstraction, ${ }^{39}$ and the molecular shape of 4-aminophenol should make it a good candidate for such an ordered arrangement with $\mathbf{R e s O}^{\bullet}$; moreover, in the case of the water-soluble vitamin $\mathrm{E}$ analogue trolox, where the value of the KIE clearly supports a direct hydrogen abstraction, the rate constant of the $\mathbf{R e s O}^{\bullet}$ repair also does not reflect the energy balance.

Finally, we focused on cysteine because that amino acid is the redox-active residue of glutathione, the only other molecule besides ascorbate and 4-aminophenol that has ever been reported to repair $\mathbf{R e s}^{\bullet}$; in experiments with $\mathbf{R e s O}^{\bullet}$ generation by $\mathrm{H}_{2} \mathrm{O}_{2}$ /horseradish peroxidase that repair was inferred from the detection of a spin-trapping adduct of the glutathionyl radical $^{21}$ and from spectroscopic observation in the UV-C. ${ }^{22}$ Our photoionization method indeed gave a transient absorption at $410 \mathrm{~nm}$ with a complex time dependence, which might mistakenly be ascribed to $\mathbf{R e s O}^{\bullet}$ alone and its repair by cysteine. However, monitoring at $650 \mathrm{~nm}$, where no cysteinederived radical interferes, afforded unambiguous evidence against a repair: on a timescale five times longer than in Fig. $2 \mathrm{~b}$, the very slow decay of the $\mathbf{R e s O}^{\bullet}$ signal proved to be completely uninfluenced by the cysteine concentration over a range from $70 \mathrm{mM}$ to $400 \mathrm{mM}$. Because the data in Table 1 indicate this repair also to be thermodynamically impossible, we suggest that the time dependence of the $410 \mathrm{~nm}$ absorption is largely due to the dimeric radical anion $\mathbf{R}-\mathbf{S}^{\bullet}-\mathbf{S}-\mathbf{R}^{-}$, which can be formed through scavenging of both $\mathrm{e}_{\mathrm{aq}}{ }^{\bullet-}$ and hydroxyl radicals by cysteine ${ }^{47}$ similar mechanisms are conceivable for glutathione, for which we found the same concentration independence.

The well-known pecking order of antioxidants describes the thermodynamic feasibility of a repair but does not allow a prediction of its precise rate, ${ }^{26}$ so by no means supersedes kinetic studies. A straightforward placement of ResOH in that sequence is hampered by the uncertainty of the standard potential for its electrochemical oxidation, which is irreversible. ${ }^{46}$ Our observation of the very fast repair of $\mathbf{R e s O}^{\bullet}$ by trolox, electrochemically an almost exact analogue of $\alpha$-tocopherol, ${ }^{26}$ complements an earlier study that gave an extremely slow rate for the reverse process, the repair of the $\alpha$-tocopherol phenoxy radical by $\mathbf{R e s O H}^{{ }^{14}}$ Together with the inertness of $\mathbf{R e s O}^{\bullet}$ against cysteine, this puts ResOH roughly halfway between these two co-antioxidants, so shows that its position in the pecking order should not deviate greatly from that given by its irreversible potential.

\section{Conclusions}

In summary, we have developed a strategy for the direct generation and isolated observation of the resveratrol phenoxy radical in the presence of co-antioxidants, an approach capable of providing detailed mechanistic and kinetic information about the fundamental, but hitherto virtually unknown repair reactions of this radical in a very straightforward manner. Our experimental procedure should be particularly attractive for studying the regeneration of the amphiphilic antioxidant resveratrol in supramolecular assemblies such as micelles, vesicles and liposomes. A second advantage can be envisaged for mixtures of resveratrol with other phenolic antioxidants, as contained in red wine and other foods; ${ }^{3}$ for such complex systems, whose interactions are largely unknown to date, the possibility of selectively accessing and monitoring the resveratrol phenoxy radical could prove very fruitful.

\section{Experimental section}

The main investigation method of this study was nanosecond laser flash photolysis with optical detection of the intermediates. A detailed description of our setup has been given elsewhere. ${ }^{38}$ 
Briefly, the collimated beam of a frequency-tripled Nd:YAG laser (Continuum Surelite-III, $355 \mathrm{~nm}, 5 \mathrm{~ns}$ pulse width) was used for homogeneous excitation of trans-resveratrol (ResOH) solutions pumped through a suprasil cell; the transient absorptions were monitored at right angle to the excitation. The absorption of the solutions at the excitation wavelength was below 0.02 , which ensured a uniform concentration of transients also in the direction of the laser beam.

All chemicals were obtained commercially in the highest available purity and used as received (trans-resveratrol, $>98 \%$, TCI; sodium ascorbate, 99\%, Alfa Aesar; trolox, 97\%, Aldrich; 4-aminophenol, $\geq 99 \%$, Aldrich; cysteine, $\geq 98.5 \%$, Aldrich; glutathione, $\geq 98 \%$, Aldrich; potassium iodide, 99\%, Aldrich; sodium dodecyl sulfate, $\geq 99.5 \%$, Roth; sodium hydrogen phosphate, $\geq 99 \%$, Fluka; sodium dihydrogen phosphate, >98\%, VEB Laborchemie Apolda). The solvent was either ultrapure Millipore MilliQ water (specific resistance, $18.2 \mathrm{M} \Omega \mathrm{cm}$ ) or-for the measurements of the $\mathrm{H} / \mathrm{D}$ kinetic isotope effects- $\mathrm{D}_{2} \mathrm{O}(99.9 \%$ deuteration, VEB Berlin Chemie).

ResOH solutions were always freshly prepared before an experiment and stored in the dark to avoid geometrical isomerization. The desired $\mathrm{pH}$ values were adjusted under pH meter control (Hanna Instruments HI 9024) by mixing sodium hydrogen phosphate and sodium dihydrogen phosphate stock solutions. The buffer concentration normally was $6.7 \mathrm{mM}$; if necessary, it was increased up to $20 \mathrm{mM}$ to keep the $\mathrm{pH}$ constant even in the presence of higher concentrations of pH-active co-antioxidants.

All solutions were purged with argon (5.0, Air Liquide) or $\mathrm{N}_{2} \mathrm{O}$ (5.0, Air Liquide) for 30 minutes before and for the whole duration of the laser flash photolysis experiments. Chemicals that are susceptible to oxidation by traces of molecular oxygen (sodium ascorbate and 4-aminophenol) were added in solid form to the already degassed solutions immediately before starting the measurements.

Steady-state absorption and fluorescence spectra were recorded with a Shimadzu UV-2102 spectrophotometer and a LS 50B spectrometer (Perkin Elmer).

\section{Acknowledgements}

Financial support provided by the Chemical Industry Funds to C.K. is gratefully acknowledged.

\section{References}

1 R. E. King, J. A. Bomser and D. B. Min, Compr. Rev. Food Sci. Food Saf., 2006, 5, 65-70.

2 J. A. Baur and D. A. Sinclair, Nat. Rev. Drug Discovery, 2006, 5, 493-506.

3 S. Quideau, D. Deffieux and L. Pouységu, Angew. Chem., Int. Ed., 2012, 51, 6824-6826.

4 C. Sánchez-Moreno, J. A Larrauri and F. Saura-Calixto, J. Sci. Food Agric., 1998, 76, 270-276.
5 B. Fauconneau, P. Waffo-Teguo, F. Huguet, L. Barrier, A. Decendit and J.-M. Merillon, Life Sci., 1997, 61, 2103-2110.

6 D. G. Soares, A. C. Andreazza and M. Salvador, J. Agric. Food Chem., 2003, 51, 1077-1080.

7 S. S. Leonard, C. Xia, B.-H. Jiang, B. Stinefelt, H. Klandorf, G. K. Harris and X. Shi, Biochem. Biophys. Res. Commun., 2003, 309, 1017-1026.

8 L. A. Stivala, M. Savio, F. Carafoli, P. Perucca, L. Bianchi, G. Maga, L. Forti, U. M. Pagnoni, A. Albini, E. Prosperi and V. Vannini, J. Biol. Chem., 2001, 276, 22586-22594.

9 R. Pulido, L. Bravo and F. Saura-Calixto, J. Agric. Food Chem., 2000, 48, 3396-3402.

10 J. S. Wright, E. R. Johnson and G. A. DiLabio, J. Am. Chem. Soc., 2001, 123, 1173-1183.

11 D. Mikulski, R. Górniak and M. Molski, Eur. J. Med. Chem., 2010, 45, 1015-1027.

12 R. Amorati, M. Lucarini, V. Mugnaini and G. F. Pedulli, J. Org. Chem., 2004, 69, 7101-7107.

13 S. Stojanović, H. Sprinz and O. Brede, Arch. Biochem. Biophys., 2001, 391, 79-89.

14 J.-G. Fang, M. Lu, Z.-H. Chen, H.-H. Zhu, Y. Li, L. Yang, L.-M. Wu and Z.-L. Liu, Chem. - Eur. J., 2002, 8, 4191-4198.

15 S. Stojanović and O. Brede, Phys. Chem. Chem. Phys., 2002, 4, 757-764.

16 D.-D. Li, R.-M. Han, R. Liang, C.-H. Chen, W. Lai, J.-P. Zhang and L. H. Skibsted, J. Phys. Chem. B, 2012, 116, 7154-7161.

17 S. Petralia, C. Spatafora, C. Tringali, M. C. Foti and S. Sortino, New J. Chem., 2004, 28, 1484-1487.

18 I. Dzeba, T. Pedzinski and B. Mihaljević, Radiat. Phys. Chem., 2012, 81, 1294-1296.

19 J. E. Packer, T. F. Slater and R. L. Wilson, Nature, 1979, 278, 737-738.

20 J. Chaudière and R. Ferrari-Iliou, Food Chem. Toxicol., 1999, 37, 949-962.

21 M. J. Burkitt and J. Duncan, Arch. Biochem. Biophys., 2000, 381, 253-263.

22 G. Galati, O. Sabzevari, J. X. Wilson and P. J. O'Brien, Toxicology, 2002, 177, 91-1043.

23 H. S. Mahal and T. Mukherjee, Res. Chem. Intermed., 2006, 32, 59-71.

24 E. Niki, A. Kawakami, Y. Yamamoto and Y. Kamiya, Bull. Chem. Soc. Jpn., 1985, 58, 1971-1975.

25 R. H. Bisby and A. W. Parker, FEBS Lett., 1991, 290, 205-208.

26 G. R. Buettner, Arch. Biochem. Biophys., 1993, 300, 535-543.

27 R. H. Bisby and A. W. Parker, Arch. Biochem. Biophys., 1995, 317, 170-178.

28 S. V. Jovanovic, C. W. Boone, S. Steenken, M. Sinoga and R. B. Kaskey, J. Am. Chem. Soc., 2001, 123, 3064-3068.

29 E. J. Land, G. Porter and E. Strachan, Trans. Faraday Soc., 1961, 57, 1885-1893.

30 J. Feitelson, E. Hayon and A. Treinin, J. Am. Chem. Soc., 1973, 95, 1025-1029.

31 J.-C. Mialocq, J. Sutton and P. Goujon, J. Chem. Phys., 1980, 72, 6338-6345.

32 S. V. Jovanovic, Y. Hara, S. Steenken and M. G. Simic, J. Am. Chem. Soc., 1995, 117, 9881-9888. 
33 A. W. Parker, R. H. Bisby, G. M. Greetham, P. Kukura, K. M. Scherer and M. Towrie, J. Phys. Chem. B, 2014, 118, 12087-12097.

34 I. Dzeba, T. Pedzinski and B. Mihaljević, J. Photochem. Photobiol., A, 2015, 299, 118-124.

35 U. Lachish, A. Shafferman and G. Stein, J. Chem. Phys., 1976, 64, 4205-4211.

36 M. Goez and C. Kerzig, Angew. Chem., Int. Ed., 2012, 51, 12606-12608.

37 M. Goez, C. Kerzig and R. Naumann, Angew. Chem., Int. Ed., 2014, 53, 9914-9916.

38 C. Kerzig and M. Goez, Phys. Chem. Chem. Phys., 2014, 16, 25342-25349.

39 W. J. Leigh, E. C. Lathioor and M. J. St. Pierre, J. Am. Chem. Soc., 1996, 118, 12339-12348.
40 J. J. Warren and J. M. Mayer, J. Am. Chem. Soc., 2010, 132, 7784-7793.

41 P. Wardman, J. Phys. Chem. Ref. Data, 1989, 18, 1637-1755.

42 F. G. Bordwell and J.-P. Cheng, J. Am. Chem. Soc., 1991, 113, 1736-1743.

43 M. Lucarini, P. Pedrielli, G. F. Pedulli, S. Cabiddu and C. Fattuoni, J. Org. Chem., 1996, 61, 9259-9263.

44 B. Fiser, M. Szori, B. Jójárt, R. Izsák, I. G. Csizmadia and B. Viskolcz, J. Phys. Chem. B, 2011, 115, 11269-11277.

45 R. Amorati, F. Ferroni, G. F. Pedulli and L. Valgimigli, J. Org. Chem., 2003, 68, 9654-9658.

46 O. Corduneanu, P. Janeiro and A. M. O. Brett, Electroanalysis, 2006, 18, 757-762.

47 M. Z. Hoffman and E. Hayon, J. Phys. Chem., 1973, 77, 990-996. 